\title{
A Near Miss Involving Cyclophosphamide
}

\author{
Contributions to this column are prepared by the Institute for Safe Medication Practices Canada (ISMP \\ Canada), a key partner in the Canadian Medication Incident and Prevention System. From time to time, \\ ISMP Canada invites others to share learning based on local initiatives. The article presented here is \\ reprinted, with permission, from the ISMP Canada Safety Bulletin 2008;8(7):1-2.
}

$\mathrm{R}$ eports of near-miss incidents offer valuable learning opportunities. ${ }^{1}$ This bulletin highlights a near miss with cyclophosphamide for intravenous administration, with the goal of sharing learning and recommendations related to the use of cytotoxic medications for non-oncology indications.

\section{MEDICATION INCIDENT}

A 55-kg adult patient was receiving care in an intensive care unit. During the patient's stay, Wegener's granulomatosis was diagnosed, and a physician ordered cyclophosphamide 2.2 grams daily, by intravenous administration, for 3 days. The pharmacist who reviewed the order checked the patient's medication profile in the pharmacy but could not identify the indication for cyclophosphamide. Furthermore, given the dose of cyclophosphamide that had been ordered, the pharmacist expected an accompanying order for the bladder-protective drug mesna, but there was no order for this drug. The pharmacist contacted the intensive care unit and was advised of the patient's new diagnosis by one of the nurses. The pharmacist initiated a literature search, because she believed that usual cyclophosphamide dosing for an autoimmune disorder such as Wegener's granulomatosis would be much lower than the dose that had been prescribed. The literature review confirmed the pharmacist's suspicions, and she contacted the physician. The physician initially affirmed the order as prescribed, but after discussing the matter further with the pharmacist and reviewing the information presented by the pharmacist, the physician realized that he had intended to order a dose of $220 \mathrm{mg}$. The physician changed the order and expressed gratitude for the pharmacist's follow-up.

\section{CONTRIBUTING FACTORS}

The following factors were identified as potentially contributing to this near-miss incident:

- The physician had intended an intravenous dose of
$4 \mathrm{mg} / \mathrm{kg}$ per day $\times 55 \mathrm{~kg}$ (the patient's body weight), for a total of $220 \mathrm{mg}$ or 0.22 grams. However, when calculating the dose, the physician misread his handwritten note about the weight-based dose. The handwritten note stated " $4.0 \mathrm{mg} / \mathrm{kg}$ ", but the physician misread the amount as " 40 $\mathrm{mg} / \mathrm{kg}$ ” and he consequently ordered 2.2 grams, instead of the intended 0.22 grams.

- Protocols for cytotoxic medications used for non-oncology indications were not readily available to either the physician who prescribed the drug or the pharmacist who checked the order. (In contrast, when a cytotoxic agent is ordered for an oncology indication, the facility requires that the most current protocol be printed from a provincial cancer website and placed in the patient's chart. These oncology protocols are publicly available and are readily accessible to all staff.)

\section{RECOMMENDATIONS}

Procedures that govern the use of cytotoxic medications for oncology indications are also applicable when medications such as cyclophosphamide are used for non-oncology purposes. This near-miss incident exemplifies the value of ensuring that all orders for cytotoxic medications are reviewed by a pharmacist with the skills for performing such reviews. The following recommendations were developed in collaboration with the reporting facility:

- Require that the order specify the therapeutic protocol being used. If a standard protocol (specific to the hospital or from another recognized source) is not readily available, require that the prescriber provide, before the cytotoxic medication is prepared, appropriate references for the specific dose and dose schedule that have been prescribed. In addition, a copy of any such reference should be included in the patient's chart, and the information should be made readily available (e.g., through sufficient additional copies or electronic access) for use by pharmacy and nursing staff. 
- Require that all orders for cytotoxic medications include the relevant patient diagnosis. Given the variety of indications for which cytotoxic agents can be used, and the resulting wide variation in doses and administration schedules, members of the care team (e.g., pharmacists and nurses) need specific diagnostic information to confirm appropriate dose ranges. Admission orders typically include the admission diagnosis, but some comorbidities may not be listed on admission. In addition, conditions diagnosed during the hospital stay may not appear in subsequent orders.

- Regardless of the indication, require that all orders for cytotoxic medications include the patient's weight (and height, if the body surface area must be calculated) to allow staff to double-check the dose ordered.

- Develop standard protocols for cytotoxic medications commonly used for non-oncology indications. Ensure that practitioners have ready access to appropriate protocols and other drug information resources. For example, at the hospital where the near miss occurred, the intravenous therapy manual has since been revised to include dosing for cyclophosphamide and other cytotoxic medications used for non-oncology indications.

- Build optimal safeguards into the ordering process for cytotoxic medications, regardless of indication. Consider incorporating quality checks into preprinted orders and electronic order entry systems, such as the following: reference dose ranges and dosing schedules, criteria for withholding or reducing the dose (e.g., threshold for absolute neutrophil count), a place to show dose calculations, and a clear indication of the day(s) on which the drug is to be given.

- Integrate predefined order sets and protocols, when possible, into computerized prescriber order entry and pharmacy information systems. Include built-in maximum-dose alerts.

- Avoid use of dangerous dose designations such as trailing zeros. $^{2}$

Cytotoxic medications are high-alert medications. ${ }^{3}$ Furthermore, cytotoxic drugs have a narrow therapeutic window (the difference between an effective dose and a toxic dose), regardless of the indication for which they are used. When cytotoxic agents are ordered for the treatment of cancer, the protocols are typically readily available, and the drugs are ordered, dispensed, and administered by specially trained health care professionals. In addition, the high-alert nature of these medications is well recognized in oncology practice, and stringent processes, including ensuring availability of the information required to process an order and performing the necessary multiple checks, are routine. Similar system-based safeguards are required for cytotoxic medications used for non-oncology indications.

\section{References}

1. Near miss identification and reporting. ISMP Can Saf Bull 2007[cited 2008 Aug 29];7(7):1-2. Available from: http://www.ismp-canada.org/ download/ISMPCSB2007-07NearMiss.pdf

2. Eliminate use of dangerous abbreviations, symbols, and dose designations. ISMP Can Saf Bull 2006[cited 2008 Oct 22];6(4): 1-3. Available from: http://www.ismp-canada.org/download/ ISMPCSB2006-04Abbr.pdf

3. ISMP's list of high-alert medications [Internet]. Huntingdon Valley (PA): Institute for Safe Medication Practices; 2008 [cited 2008 Sep 2]. Available from: http://www.ismp.org/Tools/highalertmedications.pdf

\section{Acknowledgements}

ISMP Canada gratefully acknowledges the input provided by Larry Broadfield, BScPhm, MHSc, FCSHP, Manager Systemic Therapy Program, Cancer Care Nova Scotia, and the expert review by (in alphabetical order): Patti Cornish, RPh, BScPhm, Patient Safety Service, Sunnybrook Health Sciences Centre; Roxanne Dobish, BSc(Pharm), Assistant Director, Provincial Pharmacy, Cross Cancer Institute; Robin J Ensom, PharmD, FCSHP, Regional Director, Pharmacy, Vancouver Coastal Health and Providence Health Care; Edward Etchells, MD, FRCPC, Director, Patient Safety Service, and Staff Physician, Division of General Internal Medicine, Sunnybrook Health Sciences Centre; Sophie Kim, BScPhm, Department of Pharmacy, Princess Margaret Hospital; Sharon F Lane, RN, MSN, AOCN, Associate Director Center for Patient Safety, Dana-Farber Cancer Institute; Dan Perri, BScPhm, MD, FRCPC, Clinical Pharmacologist and Intensivist, St. Joseph's Healthcare Hamilton, Assistant Professor, Department of Medicine, McMaster University; John W Senders, PhD, Professor Emeritus, University of Toronto.

Medication incidents (including near misses) can be reported to ISMP Canada in 1 of 2 ways:

- through the secure web portal at http://www.ismp-canada.org/ err_report.htm

- by telephone at 416.733 .3131 or toll-free at 1.866.544.7672 (1.866.54.ISMPC) 